\title{
Über Verbindungen des zweiwertigen Kobalts und Nickels mit Brenzkatechin. II.
}

\author{
Von R. F. Weinland und Anna Döttinger.
}

Wie wir in der ersten Abhandlung $\left.{ }^{1}\right)$ mitteilten, hatten wir gefunden, daB das zweiwertige Kobalt mit Brenzkatechin komplexe Anionen zu bilden vermag, nämlich das zweibasiche Di- und das vierbasische 'Tri-brenzkatechinato-anion, wie folgende Formeln von Verbindungen, welche diest beiden Anionen enthalten, zeigen:

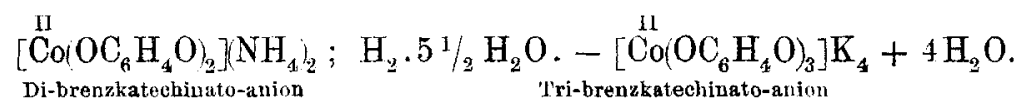

Alle diese Verbindungen zeigen die gewöhnliche rote Farbe der wasserhaltigen Kobaltosalze. Wir hatten das zuletzt angeführte Kaliumsalz nur bei ganz besonders groBen Überschuß sn Alkali erhalten (auf 1 Mol. Kobaltoacetat 40 Mol. Kaliumbydroxyd und 3 Mol. Brenzkatechin) und versuchten deshalb, anf diese Weise auch ein Natriumsalz zu bekommen. Hierbei machten wir die interessante Beobachtung, daß aus Lösungen, die sogar weniger Natriumhydroxyd enthielten, als wir tür das Kaliumsalz brauchten, ein basisches Natriumsalz auskristallisierte, in welchem auf 2 Atome Kobalt 4 Brenzkatechinreste und 7 Atome Natrium statt 4 wie beim normalen Salz, kommen:

$$
\left.2\left[\mathrm{Co}\left(\mathrm{OC}_{6} \mathrm{H}_{4} \mathrm{O}\right)_{2}\right] \mathrm{Na}_{2}+3 \mathrm{NaO}\right) \mathrm{H}+14 \mathrm{H}_{2} \mathrm{O} \text {. }
$$

Es ist zu bemerken, daß basische Natriumsalze bis jetzt nur ganz selten beobachtet worden sind, z. B. von F. MYLIUs und R. FUnK ${ }^{2}$ ) ein basisches Chromat der Formel

$$
\mathrm{Na}_{2} \mathrm{CrO}_{4}+2 \mathrm{NaOH}+12 \mathrm{H}_{2} \mathrm{O} \text {. }
$$

Dieses basische Chromat und das obige basiche Natriumsalz gehören im Sinne der Koordinationslelre zu denjenigen basi:chen Salzen, welche Anlagerungsverbindungen vorstellen, im Gegensatz zu solchen, die wie das basische Calciumchlorid und gewisse Mine-

1) Z. anorg. "r. allg. (hem. 102 (1918), 233 .

2) Ber. deutsch. chem. Ges. $\mathbf{3 3}$ (1900): 3686. 
ralien (A takamit, Alaunstein), als Einlagerunsgverbindungen angesehen werden:

$$
\begin{aligned}
& \left.\mathrm{CaCl}_{2}+3 \mathrm{Ca}(\mathrm{OH})_{2}+12 \mathrm{H}_{2} \mathrm{O}=\mid \mathrm{Ca}\left(\mathrm{Ca}(\mathrm{OH})_{2}\right)_{3}\right] \mathrm{Cl}_{2}+12 \mathrm{H}_{2} \mathrm{O} ; \\
& {\left[\mathrm{Cu}\left(\mathrm{Cu}(\mathrm{OH})_{2}\right)_{3}\right] \mathrm{Cl}_{2} ;\left[\mathrm{Al}\left(\mathrm{Al}(\mathrm{OH})_{3}\right)_{2}\right]_{\mathrm{SO}_{4}}^{\mathrm{SO}_{4}} \mathrm{~K} .}
\end{aligned}
$$

Es kommen ihnen dann folgende Formeln zu, wobei das basische Natrium-dibrenzcatechiniito-kobaltoat eine Doppelverbindung zweier Moleküle vorstellt:

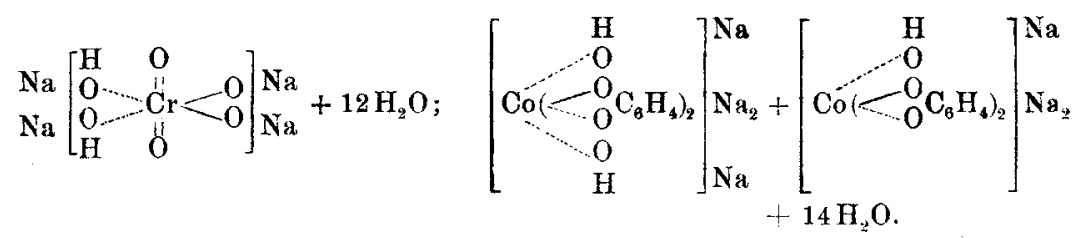

Dem letzteren Salze liegen demnach gemischte Hydroxo-brenzkatechinato-kobalto-anionen zugrunde von der Art des Dihydroxotetrachloro-plate-anions, welches in der durch Anlagerung von 2 Mol. Wasser an Platintetrachlorid entstehenden Säure der Formel

$$
\left[\mathrm{Pt}_{(\mathrm{OH})_{2}}^{\mathrm{Cl}_{4}}\right] \mathrm{H}_{2}
$$

enthalten ist. Die Koordinationszahl des Kobalts ist nach der obigen. Formulierung in dem einen Anion 6, im anderen 5. Das letztere ist aber unwahrscheinlich, man wird annehmen müssen, da B ein Wassermol. die sechste Koordinationsstelle besetzt.

Die Beobachtung dieses basichen Kobalt-brenzkatechin-natriumsalzes veranlaßte uns, zu untersuchen, ob sich ein solches basisches Salz auch beim Nickel darstellen lasse. Dies war indessen nicht der Fall. Wir erhielten auch bei sehr großem Überschuß an Natriumhydroxyd normale Salze, von denen wir die zwei folgenden in einheitlichem Zustande darstellter (s. die präparativen Finzelheiten im Versuchsteile S. 172):

$$
\begin{gathered}
{\left[\mathrm{Ni}\left(\mathrm{OC}_{6} \mathrm{H}_{4} \mathrm{O}\right)_{2}\right] \mathrm{Na}_{2}+\underset{\text { Dünne, blabgrüne Blattchen. }}{\left[\mathrm{Ni}\left(\mathrm{OC}_{6} \mathrm{H}_{4} \mathrm{O}_{3}\right] \mathrm{Na}_{4}\right.}+18 \mathrm{H}_{2} \mathrm{O} ;} \\
{\left[\mathrm{Ni}\left(\mathrm{OC}_{6} \mathrm{H}_{4} \mathrm{O}\right)_{2}\right] \mathrm{Na}_{2}+12 \mathrm{H}_{2} \mathrm{O} .} \\
\text { Dicke, dunkelgrüne Tafeln. }
\end{gathered}
$$

Die letzteren zeigten ein eigentümliches Aufblättern beim Verwittern, s. unten S. 173.

Wir hatten des weiteren in der ersten Abhandlung Anm. 1. S. 224, Brl. 102. erwähnt, daß beim Erhitzen einer Lösung von Kobaltoacetat 
und Brenzkatechin sich hellrote, pulverförmige Niederschläge abscheiden. Wir haben nunmehr fürs erste festgestellt, dab dieser rötliche Niederschlag ein basisches Kobalto-brenzkatechinat folgender Zusammensetzung

$$
\mathrm{Co}_{6}\left(\mathrm{C}_{6} \mathrm{H}_{4} \mathrm{O}_{2}\right)_{5}(\mathrm{OH})_{2} \cdot 8 \mathrm{H}_{2} \mathrm{O}
$$

ist. Es schließt sich an das früher (1. c. S. 225) beschriebene basische Kobalto-brenzkatechinat der Formel

$$
\mathrm{Co}_{3}\left(\mathrm{C}_{6} \mathrm{H}_{4} \mathrm{O}_{2}\right)_{2}(\mathrm{OH})_{2} \cdot 6 \mathrm{H}_{2} \mathrm{O}
$$

an, welches man durch Zusatz bestimmter Mengen Ammoniak zu einer Lösung von Kobaltosulfat und Brenzkatechin bekommt.

Außerdem aber beobachteten wir, daB aus sthr konzentrierten Lösungen von Kobaltoacetat und Brenzkatechin beim Erhitzen eine eigentümliche, in blauen Nadeln kristallisierende Verbindung sich ausscheidet, die gleichzeitig Brenzkatechin und Essigsäure enthält und zwar von beiden je einen Rest auf 1 Atom Kobalt. Außerdem enthält sie 1 Mol. Wasser:

$$
\mathrm{Co}\left(\mathrm{CH}_{3} \mathrm{COO}\right)\left(\mathrm{OC}{ }_{6} \mathrm{H}_{4} \mathrm{OH}\right) \cdot \mathrm{H}_{2} \mathrm{O} \text {. }
$$

Was die Konstitution dieser Verbindung betrifft, so läßt sich etwas bestimmtes darüber nicht aussagen. Wenn das Brenzkatechin, wie in den anderen Verbindungen, zwei Koordinationsstellen besetzt, und auBerdem das Wasser durch eine Nebenvalenz des Sauerstoffs an das Kobalt gebunden ist (eine andere Annahme über die Bindung les Wassers kommt kaum in Frage), würde das Kobaltatom die Koordinationszahl 4 aufweisen und man hätte es entweder mit einem Nichtelektrolyten (innerem Komplexsalz erster Ordnung) oder mit einer Säur" zu tun:

$$
\stackrel{\mathrm{CH}_{3} \mathrm{COO}}{\mathrm{H}_{2} \mathrm{O}}>\mathrm{Co}<\mathrm{O}_{6} \mathrm{C}_{4} \text { oder }\left[\begin{array}{r}
\mathrm{CH}_{3} \mathrm{COO} \\
\mathrm{H}_{2} \mathrm{O}
\end{array} \mathrm{Co}_{\mathrm{O}}^{\mathrm{O}} \mathrm{C}_{6} \mathrm{H}_{4}\right] \mathrm{H} .
$$

Für die erste Formel spricht die neue Farbe, sowie die Schwerlöslichkeit der Verbindung, welch letztere Eigenschaft bei Nichtelektrolyten häufig rorkommt.

Wir versuchten, auch in diesem Fall eine analoge Nickelverbindung darzustellen. indessen vergebens. Wir erhielten beim Frhitzen einer Lösung von Nickelacetat und Brenzkatechin nur pulverförmige, grünliche Ausscheidungen, die ein basisches Nickelbrenzkatechinat vorstellen, das den roten basischen Kobalt-brenz- 
katechinaten (s. oben S. 169) an die Seite zu stellen ist und folgende Zusammensetzung hat:

$$
\mathrm{Ni}_{3}\left(\mathrm{C}_{6} \mathrm{H}_{4} \mathrm{O}_{2}\right)_{2}(\mathrm{OH})_{2} .5 \mathrm{H}_{2} \mathrm{O} \text {. }
$$

Versuchsteil.

1. Basisches Natriumsalz der Di-brenzatechinato.

kobaltosäure.

(Vergleiche oben S. 167.)

Man löst $2.5 \mathrm{~g}$ Kobaltacetat unter Frwärmen in $8 \mathrm{ccm}$ Wasser, fügt $3.3 \mathrm{~g}$ Brenzkatechin hinzı und sodann eine heiße Lösung von $8 \mathrm{~g}$ Natriumbydroxyd in $8 \mathrm{~g}$ Wasser (1 Co: 8 Brenz.: $20 \mathrm{NaOH}$ ). Es scheidet sich zunächst ein blaßroter, flockiger Niederschlag aus, der sich beim Zusatz der übrigen Menge Natronlauge wieder löst. Die heiBe Lösung ist blau, beim Erkalten wird sie tiefrot. Aus dieser Lösung kristallisiert im Laufe einiger Stunden das in der Übersehrift genannte Natriumsalz aus. Man gießt die Mutterlauge sorgfältig ab, wäscht die Kristalle einigemale dekantierend mit eiskaltem Wasser und trocknet sie über Chlorcalcium.

Das Salz bildet dünne, rote, schmale, schief oder dachförmig begrenzte Tafeln und ist in Wasser sehr leicht löslich mit roter Farbe. An der Luft färbt es sich dunkel.

$$
\begin{aligned}
& \text { Analyse. } \\
& 0.5333 \mathrm{~g} \text { Sulst.: } 0.0627 \mathrm{~g} \text { ( } 0 . \cdots-0.4799 \mathrm{~g} \text { Subst.: } 0.0053 \mathrm{~g} \text { Co. } \\
& 0.5154 \mathrm{~g} \quad . . \quad 0.0600 \mathrm{~g}(\mathrm{C} . \quad-0.5119 \mathrm{~g} \text { Subst.: } 0.0592 \mathrm{~g} \mathrm{Co} .
\end{aligned}
$$

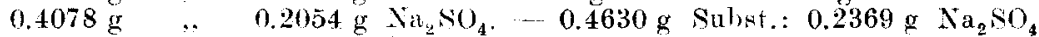

$$
\begin{aligned}
& 0.1551 \mathrm{~g} \quad, . \quad 0.1657 \mathrm{~g} \mathrm{CO}_{2}, 0.0647 \mathrm{~g} \mathrm{H} \mathrm{H}_{2} \mathrm{O} .- \\
& 0.1351 \mathrm{~g} \quad, .0 .1415 \mathrm{~g} \mathrm{CO} \text {. - } \\
& 0.1847 \mathrm{~g} \quad, \quad 0.1953 \mathrm{~g} \quad \mathrm{CO}_{2}^{2} .0 .0794 \mathrm{~g} \mathrm{H} \mathrm{H}_{2} \mathrm{O} . \cdots \\
& 0.1676 \mathrm{~g} \quad, \quad 0.1787 \mathrm{~g}\left(\mathrm{O}_{2}\right. \text {. } \\
& 2\left[\mathrm{Co}_{6}\left(\mathrm{O}_{6} \mathrm{H}_{4} \mathrm{O}\right)_{2}\right] \mathrm{Na}_{2}+3 \mathrm{NaOH}+14 \mathrm{H}_{2} \mathrm{O}(1014.3) \text {. } \\
& \text { Ber. ('n 11.62, Na 15.87, C } 28.39 \text {, H 4.67 } \\
& \text { Gef., 11.8. .. 16.3, , 29.1, , } 4.7 \\
& \begin{array}{lllllll} 
& 11.5, & . & 16.6, & . . & 28.6, \quad " 4.7
\end{array}
\end{aligned}
$$

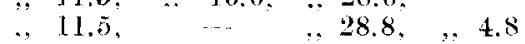

$$
\begin{aligned}
& \because 11.6, \quad-\quad, 29.1, \quad-4 .
\end{aligned}
$$

Die Werte für Na und $C$ sind etwas zu hoch; dies wird einer kleinen Henge angetrockneten Natriumearonats zuzusehreihen sein.

2. Basisches Kobalt-brenzkatechinat aus Kobaltacetat und Brenzkatechin.

(Vergleiche oben S. 169.)

Varı löst $5 \mathrm{~g}$ Kobaltacetat und $4.4 \mathrm{~g}$ Brenzkatechin in $30 \mathrm{ccm}$ Wasser (1 Co: 2 Brenzk.) und erwärmt die Lösung mehrere Stunden 
auf dem Wasserbade. Hierbei scheidet sich allmählich das in der Uherschrift genannte basische Salz in Form eines blaroten Niederschlags aus. Man wäscht es dekantierend mit kaltem Wasser, streicht auf Ton und trocknet über Chlorcalcium. Dif Ausbente ist schlecht. Das Salz bildet frisch dargestellt ein blabrotes Pulver, doch färbt es sich an der Juft raseh dunkel. In Wasser ist es schwer löslich.

$$
\begin{aligned}
& \text { An a y } y \text { se. } \\
& 0.3758 \mathrm{~g} \text { Nulst.: 0.1245 g (o. - } 0.2855 \mathrm{~g} \text { Sulst.: } 0.0957 \mathrm{~g} \text { ('o. - - } \\
& 0.1548 \mathrm{~g} \quad, \quad 0.1859 \mathrm{~g} \mathrm{CO} .0 .0466 \mathrm{~g} \mathrm{H} \mathrm{H}_{2} \mathrm{O} . \cdots \\
& 0.1817 \mathrm{~g} \quad, \quad 0.2226 \mathrm{~g} \mathrm{CO}_{2}^{-} \text {. } \\
& \left(\mathrm{CO}_{8}\left(\mathrm{C}_{6} \mathrm{H}_{4} \mathrm{O}_{2}\right)_{3}(\mathrm{OH})_{2} .8 \mathrm{H}_{2} \mathrm{O}(1072.1)\right. \text {. } \\
& \text { Ber. ('o 33.0, (' 33.58, H } 3.57 \\
& \text { Gef. .. 333.1, .. 33.7. .. } 3.3 \\
& \text {, } 33.5 . \quad \text {. } 33.4 .
\end{aligned}
$$

3. Blaue Kobalt-brenzkatechin-essigsäure-Terbindung.

(Vergleiche oben \$. 169.)

Man löst $4 \mathrm{~g}$ Kobaltacetat unter Erwärmen in $5 \mathrm{ccm}$ Wasser und in dieser Iösung $4.4 \mathrm{~g}$ Brenzkatechin (1 Co zu etwa 2 Brenzk.). Die so erhaltene Lösung wird etwa 2 Stunden lang auf dern Wasserbade erwärmt, wobei die in Rede stehende Verbindung auskristallisiert. Sie wird einigemale dekantierend gewaschen und äber Chlorcalcium getrocknet. Die Ausbeute ist schlecht.

Sehr schöne blane Nadeln, in Wasser ziemlich schwer löslich. Bei längerem Aufbewahren verschwindet die schöne Farbe. Beim Kochen mit Wasser zersetzt sich die Verbindung unter Graufärbung.

$$
\text { An:Ly:ce. }
$$

$0.5190 \mathrm{~g}$ Subst.: $0.1257 \mathrm{~g}(6,-0.5647 \mathrm{~g}$ sulst.: $0.1353 \mathrm{~g}$ ('o.

$0.3171 \mathrm{~g}$ Sulst.: $0.0772 \mathrm{~g}$ ('o. $0.0954 \mathrm{~g}$ subst.: $0.1385 \mathrm{~g} \quad \mathrm{CO}_{2}, 0.0360 \mathrm{~g} \mathrm{H}_{2} \mathrm{O}$

$$
\begin{aligned}
& \left({ }^{\circ} \mathrm{O}\left(\mathrm{OC}_{6} \mathrm{H}_{4} \mathrm{OH}\right)\left({ }^{\circ} \mathrm{H}_{3} \mathrm{COO}\right) \cdot \mathrm{H}_{2} \mathrm{O}(245,0)\right. \text {. } \\
& \text { Ber. Co 24.07, ( 39.18. H } 4.11 \\
& \text { (xef. , 24.22, ,39.19, .. 4.3\% } \\
& ., 23.96 \\
& \therefore 24.34
\end{aligned}
$$

\section{Basisches Nickel-brenzkatechinat.}

(Vergleiche oben S. 169.)

Man löst $5 \mathrm{~g}$ Nickelacetat $\left(\mathrm{Ni}\left(\mathrm{CH}_{3} \mathrm{COO}\right)_{2}+4 \mathrm{H}_{2} \mathrm{O}\right)$ und $4.4 \mathrm{~g}$ Brenzkatechin (1 Ni:2 Brenzk.) in $75 \mathrm{~cm}$ Wasser und erhitzt auf dem Wasserbade. Nach mehreren Stunden gießt man die Mutterlange von dem entstandenen hellgrünen Niederschlag ab und wäscht diesen dekantierend. Da er änBerst feinkörnig ist. saugt man ihn am besten anf einem Neubauertiegel ab. Man trocknet ihn über Chlor- 
calcium. Frisch dargestellt bildet die Verbindung ein feines, hellgrünes Pulver. Sie färbt sich aber an der Luft bald grau. In Wasser ist das Salz schwer löslich.

$$
\text { Analyoe. }
$$

$0.2584 \mathrm{~g}$ Subst.: $0.0888 \mathrm{~g} \mathrm{Ni.1)} \mathrm{-} 0.2099 \mathrm{~g}$ Subst.: $0.0711 \mathrm{~g} \mathrm{Xi.}$

$0.1745 \mathrm{~g}$ Subst.: $0.1784 \mathrm{~g}\left(\mathrm{O}_{2}, \ldots .1286 \mathrm{~g}\right.$ Subst.: $0.1294 \mathrm{~g} \mathrm{CO}_{2}$.

$$
\begin{aligned}
& \mathrm{Ni}_{3}\left(\mathrm{C}_{6} \mathrm{H}_{4} \mathrm{O}_{2}\right)_{2}(\mathrm{OH})_{2} \text {. } 5 \mathrm{H}_{2} \mathrm{O} \text { (516.2). } \\
& \text { Ber. Ni 33.71, ('27.89 } \\
& \text { Gef. , 34.t. ,. } 27.9 \\
& .33 .9 . \quad .27 .4
\end{aligned}
$$

5. Natrium-di- and tri-bren\%katechinato-nickeloat.

(Vergleiche oben S. 168.)

Man löst $1.25 \mathrm{~g}$ Nickelacetat unter Erwärmen in $12 \mathrm{ccm}$ Alkohol und in dieser Lösung $1.65 \mathrm{~g}$ Brenzkatechin, worauf man eine Lösuug von $4 \mathrm{~g}$ Vatriumhydroxyd in $8 \mathrm{~cm}$ Wasser hinzufügt $1 \mathrm{Ni}: 3$ Brenzk: $20 \mathrm{NaOH}$ ). Es entsteht sogleich ein grülicher, amorpher, fast die ganze Flüssigkeit erfüllender Niederschlag. Dieser verschwindet allmählich in der Flüssigkeit, die man verschlossen stehen läßt, und es bilden sich im Laufe von 1--2 Tagen schiefwinklige, dünne, blaBgrüne Blättchen. Man wäscht sie dekantierend mit einer eiskalten Mischung glejcher Raumteile Wasser und Alkohol, streicht auf Ton und trocknet über Chlorcalcium. Das Salz ist in Wasser sehr leicht löslich und färbt sich an der Iuft rasch gran.

\section{Analyse.}

$0.5169 \mathrm{~g}$ Subst.: $0.2742 \mathrm{~g}$ Dimethylglyoxim-nickel und $0.1963 \mathrm{~g} \mathrm{Na}_{2} \mathrm{SO}_{4}$. - ... $0.4795 \mathrm{~g}$ Subst.: $0.0504 \mathrm{~g} \mathrm{Ni}$. $\cdots .4808 \mathrm{~g}$ Subst.: $0.1834 \mathrm{~g} \mathrm{Na}_{2} \mathrm{SO}_{4}$

$0.1650 \mathrm{~g}$ Subst.: $0.1910 \mathrm{~g} \mathrm{CO}_{2} .-0.1597 \mathrm{~g}$ Subst.: $0.1863 \mathrm{~g} \mathrm{CO}_{2}$. $\left[\mathrm{Ni}\left(\mathrm{C}_{6} \mathrm{H}_{4} \mathrm{O}_{2}\right)_{2}\right] \mathrm{Na}_{2}+\left[\mathrm{Ni}\left(\mathrm{C}_{6} \mathrm{H}_{4} \mathrm{O}_{2}\right)_{3}\right] \mathrm{Na}_{4}+18 \mathrm{H}_{2} \mathrm{O}(1119.8)$.

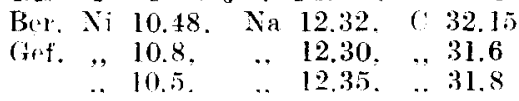

6. Natrium-di-brenzkatechinato-nickeloat.

(Vergleiche oben S. 168.)

Man löst $1.25 \mathrm{~g}$ Nickelacetat unter Erwärmen in $8 \mathrm{ccm}$ Wasser und in dieser Juösung $1.65 \mathrm{~g}$ Brenzkatechin. Dazu fügt man eine Lösung von $4 \mathrm{~g}$ Natriumhydroxyd in $8 \mathrm{cem}$ Wasser (1 Ni: 8 Brenzk. : $20 \mathrm{NaOH}$ ). Es entsteht sofort eine amorphe, grünliche Fällung, die im Laufe weniger Stunden verschwindet, während sich die

1) Ni elektrisch abgeschieden, wie Co, s. Z anorg. Chem. 102 (1918), 234. 
Flüssigkeit tief dunkel färbt. Im Laufe einiger Tage scheilet sich aus ihr das in Rede stehende salz in einzelnen, dickplattigen Kristallen aus. Man wäscht sie sorgfältig mit kaltem Wasser und trocknet sie äber Chlorcalcium unter Beobachtung, da sie rasch verwittern.

Das Salz bildet dicke, dunkelgrün 'lafeln, die beim Verwittern in eigentümlicher Weise aufblätern. Herr Prof. Dr. P. Nigan hatte die Güte, die Kristalle im Mineralogischen Institut der Universität llübingen durch therm Dr. P. J. Beger, Assistenten dastlbst, untrisuchen zu lassen. Wir sagen auch an dieser Stelle beiden Herren hierfür unsern besten Dank. Herr 1or. BEakn berichtet:

.Monoklin prismatisch

$a: b=1.19: 1 ; \beta=112.5^{\circ}$;

c bloibt offen mangels ciner diese Achse schneidenden Fläche.

Beobachtete Formen $\{110\}$ und $\{001\}$, davon $\{110\}$ etwas verrundet, so daß die Winkelmossungen zur Bestimmung des Achsenverhältnisses unter dem Mikroskope vorgenommen werden muBten. Grünlichschwarz und matt glasglänzend, mehr oder weniger tafelig nach \{00l\}.

Spaltbarkeit. nach \{001\} höchst vollkommen, der des Glimmers nicht nachstehend; vollkommen |f (wahrscheinlich nach $\{100\}$ ); gut \|l $a$ (wahr. scheinlich nach $\{010\})$. Alle drei spaltbarkeiten worden durch die rasch Platz greifende Verwitterung des Salzes verbessert, die nach $\{001\}$ derart, daß Zerfall durch Aufblätern innerhalb weniger stunden eintritt.

Optische Orientierung. $\left.{ }^{1}\right)$

$c: c=2$ bis $2.5^{\circ}$ im stumpfen Winkel $\beta$.

$b=a$.

Pleohroismus kaum wabrnehmbar, immerhin erscheint $b$ etwas dunkler grün als a.

Absorption $\mathfrak{a}<\mathfrak{b}$.

Brechungsexponenten für Natriumlicht:

$$
\begin{aligned}
& \left.\begin{array}{l}
x=1.482 \\
\beta^{\prime}=1.547
\end{array}\right\} \text { gemessen. } \quad \beta-\alpha=0.065 \quad y-a=0.156 \\
& \gamma=1.638 \text { berechnet. } \quad \gamma-\beta=0.091
\end{aligned}
$$

In Wasser ist das Salz leicht löslich mit grüner Farbe.

1) Infolge der vorzüglichen spaltbarkejt nach [001] konnte die optische Untersuchung nur an Basisspaltblättchen vorgenommen werden. Daher entspricht der als $\beta$ angeführte Wert eigentlich dem der kristallographischen Achse a. $\gamma$ mußte berechnet werden. 
174 R. F. Weinland u. A. Döttinger. Verbindungen des xweiwert. Kobalts usw.

Analyse.

$0.5290 \mathrm{~g}$ Subst.: $0.2806 \mathrm{~g}$ Dimethylglyoxim-Nickel und $0.1529 \mathrm{~g} \mathrm{Na}_{2} \mathrm{SO}_{4}$.$0.5241 \mathrm{~g} \quad, \quad 0: 2870 \mathrm{~g}$ Dimethylglyoxim-Nickel und $0.1586 \mathrm{~g} \mathrm{Na} \mathrm{NO}_{4}$.-. $0.1981 \mathrm{~g} \quad " \quad 0.1847 \mathrm{~g} \mathrm{CO}_{2}$ und $0.1035 \mathrm{~g} \mathrm{H}_{2} \mathrm{O}$.

$0.2018 \mathrm{~g} \quad, \quad 0.1909 \mathrm{~g} \mathrm{CO}_{2}$ und $0.1042 \mathrm{~g} \mathrm{H}_{2} \mathrm{O}$.

$$
\left[\mathrm{Ni}\left(\mathrm{C}_{6} \mathrm{H}_{4} \mathrm{O}_{2}\right)_{2}\right] \mathrm{Na}_{2}+12 \mathrm{H}_{2} \mathrm{O}(536.9) \text {. }
$$

Ber. Ni 10.93, Na 8.56, C 26.82, H 6.0

Gef. " 10.8, "9.3, ,25.4, , 5.8

"11.1, " $9.8, \quad$, 25.8, ", 5.8

Die Werte für das Natrium sind zu hoch, die für den Kohlenstoff zu niedrig. Aus den für das Natrium gefundenen. Prozenten ergibt sich das Verhältnis $\mathrm{Ni}: \mathrm{Na}=1.0: 2.25$. Diesen zu hohen Gehalt an Natrium wird man durch etwas angetrocknete Mutterlauge erklären müssen. Die unter dieser Annahme für $\left[\mathrm{Ni}\left(\mathrm{C}_{6} \mathrm{H}_{4} \mathrm{O}_{2}\right)_{2}\right] \mathrm{Na}_{2}+0.25 \mathrm{NaOH}+12 \mathrm{H}_{2} \mathrm{O}$ berechneten Werte, nämlich $10.73 \% \mathrm{Ni}, 9.33 \% \mathrm{Na}, 26.30 \% \mathrm{C}$ und $5.90 \% \mathrm{H}$, stimmen mit den gefundenen leidlich überein. Wir möchten nicht annehmen, daß es sich um ein basisches Salz handelt, wie wir eines beim Kobalt gefunden habén, s. oben S. 167 .

Tübingen, Chem. Laboratorium der Universität, 25. Febr. 1920.

- Bei der Redaktion eingegangeu am 1. März 1920. 